\title{
Predicting Finger-Touch Accuracy Based on the Dual Gaussian Distribution Model
}

\author{
Xiaojun Bi Shumin Zhai \\ Google Inc. \\ Mountain View, CA, USA \\ \{xiaojunbi, zhai\}@acm.org
}

\begin{abstract}
Modelling the accuracy of finger-touch target acquisition is crucial for designing touchscreen UI and for modeling more complex and higher level touch interaction behaviors. Despite its importance, there has been little theoretical work on creating such models. Building on the Dual Gaussian Distribution Model[3], we derived an accuracy model that predicts the success rate of target acquisition based on the target size. We evaluated the model by comparing the predicted success rates with empirical measures for three types of targets: 1-dimensional vertical, 1-dimensional horizontal, and 2-dimensional targets. The predictions matched the empirical data very well: the differences between predicted and observed success rates were under $5 \%$ for $4.8 \mathrm{~mm}$ and $7.2 \mathrm{~mm}$ targets, and under $10 \%$ for $2.4 \mathrm{~mm}$ targets. The evaluation results suggest that our simple model can reliably predict touch accuracy.
\end{abstract}

\section{Author Keywords}

Finger touch; target acquisition; modeling

\section{ACM Classification Keywords}

H.5.2. [Information interfaces and presentation]: User Interfaces-Theory and methods.

\section{INTRODUCTION}

Acquiring targets with finger touch is one of the most important and frequent tasks on today's mobile computing devices. However, because a finger is less precise than a mouse pointer or a stylus as an input tool, finger touch is more constraining to the graphical UI design. One major difference between the current touchscreen UI and the traditional GUI designed for the mouse and keyboard input paradigm is that selectable widgets such as buttons, bars and icons have to be made much bigger on touchscreen devices to accommodate the imprecision of the finger. For example, the Android UI design guideline recommends $48 \mathrm{dp}$ (7.62 $\mathrm{mm})$ as the minimum height of a selectable widget primarily to ensure the accuracy of target selection [1].

Permission to make digital or hard copies of part or all of this work for personal or classroom use is granted without fee provided that copies are not made or distributed for profit or commercial advantage and that copies bear this notice and the full citation on the first page. Copyrights for third-party components of this work must be honored. For all other uses, contact the owner/author(s). Copyright is held by the author/owner(s).

UIST 2016, October 16-19, 2016, Tokyo, Japan.

ACM ISBN 978-1-4503-4189-9/16/10.

http://dx.doi.org/10.1145/2984511.2984546
Choosing the appropriate size for a widget is a balance between accuracy and space. On the one hand, the widgets should be big enough to accommodate the imprecision of the finger. On the other hand, big widgets occupy large screen real estate, limiting the number of widgets displayed on a screen and refraining designers from freely placing widgets. Hybrid devices which are simultaneously designed for finger, stylus and mouse input adds another layer of complexity. A mouse- or stylus-friendly design with small-sized widgets is error-prone for finger touch, whereas a finger-friendly design with big and sparse widgets results in unnecessary and long travel distances for mouse and stylus, often over separate virtual screens. UI designers need to strike a balance between these two styles.

To choose appropriate widget size, it is crucial to quantitatively understand how the target size affects user's selection accuracy. For example, UI designers often encounter the following questions. How much accuracy degradation a user might suffer if the size of a menu item decreases from $48 \mathrm{dp}$ $(7.62 \mathrm{~mm})$ to $38 \mathrm{dp}(6.03 \mathrm{~mm})$ ? If there is only $5 \mathrm{~mm} \times 5$ $\mathrm{mm}$ space, is it big enough for a radio button? An accuracy model that can predict the success rate of finger touch can not only answer these practical questions, allowing UI designers to assess design alternatives without conducting costly user studies, but also guide designers towards a touch-friendly interface at an early design stage. Additionally, such a model combined with other human behavioral models (e.g., Fitts' Law [5] and Hick's Law [8]) lays a foundation for modeling and predicting higher level user interaction performance. Furthermore, quantitative models play an essential role in automatic interface generation tools [11]. A model quantifying the precision of finger touch is vital for these tools to generate UI appropriate for mobile devices. However, as important as mobile computing has become, no quantitative UI model predicting the precision of finger touch has been offered in the UI research field.

In this paper, we propose a model that predicts the success rate of finger-touch target acquisition simply based on the target size. A user study showed that the prediction matched with empirical measures well. The differences between the predicted and observed success rates were under 5\% for 4.8 $\mathrm{mm}$ and $7.2 \mathrm{~mm}$ targets, and under $10 \%$ for $2.4 \mathrm{~mm}$ targets.

\section{RELATED WORK}

Our accuracy model is derived from the Dual Gaussian Distribution Model [3,4] in which the randomness of the touch 
point location is conceptualized as two components: one from the input finger's intrinsic ambiguity (referred as an absolute component) and the other related to the target size (referred as an relative component). Empirical evidence shows that the Dual Gaussian Distribution Model leads to improvements in modeling reciprocal target acquisition tasks (i.e., Fitts law tasks)[3] and in determining the intended target in target acquisition tasks[4]. Our accuracy prediction model is another application of the Dual Gaussian Distribution Model.

In addition to [3, 4], there has been a sizable amount of research studying finger touch as an input modality, which has advanced our understanding of this input modality and also serves as a foundation of the current work. Ample evidence exists that touch points follow bivariate Gaussian distributions in target acquisition tasks $[2,7,6,13,9,10]$. Previous research further revealed the properties of the distributions. Azenkot \& Zhai [2] and Henze et al. [6, 7] studied a special target acquisition task: entering text on a touchscreen keyboard. Their studies showed that the means of the Gaussian distributions were close to key centers, but often with a small bias in different directions depending on hand posture (index finger vs. thumb), and regions of the keyboard. Holz and Baudisch $[9,10]$ showed that users relied on the visual feature of fingers such as finger and nail outlines for placing the touch point, and the angle between the finger and touch surface (i.e., pitch, roll and yaw) affected the offsets of touch point locations from the intended point.

To cope with the ambiguity in input modalities (e.g., finger touch, speech input), researchers $[14,12]$ have also proposed general frameworks for handling uncertain input events. Our accuracy model will play an important role in such frameworks, especially for handling touch input.

It has long been recognized that accuracy (or error) models predicting the precision of human actions are important in designing and evaluating user interface. One of the resent advances is Wobbrock et al.'s work [15] which has derived an error model from Fitts' law[5] to predict the precision of reciprocal 1-dimensional pointing task for mouse and stylus input. However, despite the unequivocal importance of finger touch, there has been little work on creating models for predicting finger touch accuracy.

\section{PREDICTING ACCURACY WITH THE DUAL GAUSSIAN DISTRIBUTION MODEL}

\section{Off-Screen-Start Target Acquisition}

Depending on the starting position of the input finger, target acquisition tasks can be categorized as off-screen-start or on-screen-start. Off-screen-start means the input finger starts from a position outside the touch screen and lands on the touch screen from the space above. Common examples include using the input finger which originally rests outside the screen to click a hyperlink or an application icon on the touchscreen. In contrast, on-screen-start means the input finger starts from a position on the touch screen and travels a certain distance parallel to the touch screen to acquire the target in, for example, typing text on a touchscreen keyboard with a single finger. This research focuses on the off-screenstart target acquisition.

\section{Probability of Successful Acquisition}

The common criterion of target selection evaluates whether the touch point falls within the boundaries of the target. Under this criterion, the success rate of target acquisition is equivalent to the probability of touch points falling within the target boundaries. Since touch points follow a bivariate Gaussian distribution, its probability density function can be expressed as:

$$
P(x, y)=\frac{1}{2 \pi \sigma_{x} \sigma_{y} \sqrt{1-\rho^{2}}} \exp \left[-\frac{z}{2\left(1-\rho^{2}\right)}\right],
$$

where

$$
z \equiv \frac{\left(x-\mu_{x}\right)^{2}}{\sigma_{x}^{2}}-\frac{2 \rho\left(x-\mu_{x}\right)\left(y-\mu_{y}\right)}{\sigma_{x} \sigma_{y}}+\frac{\left(y-\mu_{y}\right)^{2}}{\sigma_{y}^{2}}
$$

and $\rho$ is the correlation of $x$ and $y .\left(\mu_{x}, \mu_{y}\right)$ is the center of the distributions. $\sigma_{x}$ and $\sigma_{y}$ are standard deviations in $x$ and $y$ directions, respectively.

The probability of successfully acquiring a target $D$ can be calculated as:

$$
P(D)=\iint_{D} P(x, y) d x d y
$$

For one dimensional targets (e.g., hyperlinks or bars), the touch pints follow a 1-dimensional Gaussian distribution, whose probability density function is:

$$
P(x)=\frac{1}{\sigma \sqrt{2 \pi}} e^{-(x-\mu)^{2} / 2 \sigma^{2}}
$$

The probability of successfully acquiring a target with two boundaries at $x_{1}$ and $x_{2}$, where $x_{1} \leqslant x_{2}$, equals to the probability of touch points falling between the boundaries:

$$
\begin{aligned}
P\left(x_{1} \leqslant\right. & \left.X \leqslant x_{2}\right)=\int_{x_{1}}^{x_{2}} P(x) d x \\
& =\frac{1}{2}\left[\operatorname{erf}\left(\frac{x_{2}-\mu}{\sigma \sqrt{2}}\right)-\operatorname{erf}\left(\frac{x_{1}-\mu}{\sigma \sqrt{2}}\right)\right]
\end{aligned}
$$

\section{Parameters of Gaussian Distribution}

Applying Equations (3) and (5) to predict the accuracy of finger touch requires the means $(\mu)$ and variances $\left(\sigma^{2}\right)$ of touch point distributions. Here is how the Dual Gaussian Distribution Model[3, 4] comes into play. We apply this model to predict the variance of the touch point distribution based on the target size.

The Dual Gaussian Distribution Model[3, 4] conceptually breaks the variance of a touch point distribution $\left(\sigma^{2}\right)$ into an absolute component $\left(\sigma_{a}^{2}\right)$ determined by the intrinsic ambiguity of the input instrument (e.g., input finger for touch interaction), and a relative component $\left(\sigma_{r}^{2}\right)$ governed by the width (or height or diameter) of the target. Most importantly, the Dual Gaussian Distribution Model implies that the variance of the distribution $\left(\sigma^{2}\right)$ has a linear relationship with the 
squared size of the target:

$$
\sigma^{2}=\sigma_{r}^{2}+\sigma_{a}^{2}=\alpha W^{2}+\sigma_{a}^{2}
$$

where $W$ is the target size. Both $\alpha$ and $\sigma_{a}^{2}$ can be determined by empirical data. We employ the parameters reported in $\mathrm{Bi}$ and Zhai's work [4]:

$$
\begin{aligned}
& \sigma_{x}^{2}=0.0075 W_{x}^{2}+1.68 \\
& \sigma_{y}^{2}=0.0108 W_{y}^{2}+1.33
\end{aligned}
$$

where $W_{x}$ and $W_{y}$ are the lengths ( $\mathrm{mm}$ ) of the target in $x$ and $y$ directions, and $\sigma_{x}^{2}$ and $\sigma_{y}^{2}$ are variance $\left(\mathrm{mm}^{2}\right)$ in $x$ and $y$ directions, respectively. For a circular target, $W_{x}=W_{y}=d$. $d$ is the target diameter.

Note that the parameters in Equations (7) and (8) are generic for input fingers (referred as generic parameters hereafter), which are averaged values across different finger types. $\mathrm{Bi}$ and Zhai [4] also reported $\sigma$ and $\alpha$ for thumb and index finger specifically. As shown in Table 1 , the differences between different types of parameters are small. By default, we use generic parameters.

\begin{tabular}{|c|c|c|c|c|}
\hline Type & $\alpha_{x}$ & $\sigma_{a_{x}}$ & $\alpha_{y}$ & $\sigma_{a_{y}}$ \\
\hline Generic & 0.0075 & 1.296 & 0.0108 & 1.153 \\
\hline Index Finger & 0.0075 & 1.241 & 0.0104 & 1.118 \\
\hline Thumb & 0.0073 & 1.349 & 0.0113 & 1.179 \\
\hline
\end{tabular}

Table 1. Empirically determined parameters for Equation (6) from Bi and Zhai's work[4]. The unit is $\mathrm{mm}$. We use the generic parameters in Equations (7) and (8) by default.

Previous research $[2,6,7]$ shows that the mean of the distribution has a small offset from the center of the target. However, no research has shown any systematic or fixed mean offset in general acquisition tasks. In fact, previous research has shown both the direction and magnitude of the offset vary depending on various factors such as the location on the screen $[6,2]$, input angle [10], etc. Since the average offset across different conditions is close to the target center [6], we assume that the mean is at the center of the target as a model simplification:

$$
\begin{aligned}
& \mu_{x}=0 \\
& \mu_{y}=0
\end{aligned}
$$

Note that if more task context such as the target location or the finger input angle is known, a more accurate model for predicting the mean value should be used.

Similarly, previous work [2] shows that correlation between $x$ and $y$ coordinates of the touch points is very small. We assume:

$$
\rho=0 \text {. }
$$

\section{Predicting Accuracy of Finger Touch}

Equations (7), (8), (9), (10), and (11) estimate the parameters necessary to describe the distributions of touch points. With this information, it is straightforward to apply Equations (3) and (5) to predict touch accuracy.
Predicting Success Rate of Acquiring 2-Dimensional Targets

Substituting $\sigma_{x}, \sigma_{y}, \mu_{x}, \mu_{y}$ and $\rho$ in the 2-dimensional probably density function (Equation (1)) with estimation in Equations (7), (8), (9), (10), and (11) yields

$$
P(x, y)=\frac{1}{2 \pi \sigma_{x} \sigma_{y}} \exp \left(-\frac{x^{2}}{2 \sigma_{x}^{2}}-\frac{y^{2}}{2 \sigma_{y}^{2}}\right),
$$

where

$$
\begin{aligned}
& \sigma_{x}=\sqrt{0.0075 W_{x}^{2}+1.68} \\
& \sigma_{y}=\sqrt{0.0108 W_{y}^{2}+1.33}
\end{aligned}
$$

Substituting $P(x, y)$ in Equation (3) with Equation (12), the success rate of acquiring a 2-dimensional target $D$ can be expressed as:

$$
\begin{aligned}
P(D) & =\iint_{D} P(x, y) d x d y \\
& =\iint_{D} \frac{1}{2 \pi \sigma_{x} \sigma_{y}} \exp \left(-\frac{x^{2}}{2 \sigma_{x}^{2}}-\frac{y^{2}}{2 \sigma_{y}^{2}}\right) d x d y
\end{aligned}
$$

where $\sigma_{x}$ and $\sigma_{y}$ can be calculated from Equations (13) and (14).

Predicting Success Rate of Acquiring 1-Dimensional Targets Similarly, substituting $\sigma$ and $\mu$ in Equation (5) which predicts the success rate of acquiring a 1-dimensional target, with estimation in Equations (7), (8), (9), and (10) leads to

$$
\begin{gathered}
P\left(x_{1} \leqslant X \leqslant x_{2}\right)=\frac{1}{2}\left[\operatorname{erf}\left(\frac{x_{2}-\mu}{\sigma \sqrt{2}}\right)-\operatorname{erf}\left(\frac{x_{1}-\mu}{\sigma \sqrt{2}}\right)\right] \\
=\frac{1}{2}\left[\operatorname{erf}\left(\frac{x_{2}}{\sigma \sqrt{2}}\right)-\operatorname{erf}\left(\frac{x_{1}}{\sigma \sqrt{2}}\right)\right] .
\end{gathered}
$$

Assuming the origin is the centroid of the target and the width or height of the target is $W$, Equation (16) becomes:

$$
\begin{aligned}
P\left(-\frac{W}{2} \leqslant X \leqslant \frac{W}{2}\right) & =\frac{1}{2}\left[\operatorname{erf}\left(\frac{W}{2 \sqrt{2} \sigma}\right)-\operatorname{erf}\left(\frac{W}{-2 \sqrt{2} \sigma}\right)\right] \\
& =\operatorname{erf}\left(\frac{W}{2 \sqrt{2} \sigma}\right) .
\end{aligned}
$$

Note that in both Equations (16), and (17), $\sigma=\sigma_{x}$ for vertical targets and $\sigma=\sigma_{y}$ for horizontal targets. $\sigma_{x}$ and $\sigma_{y}$ are calculated in Equations (13) and (14).

Equations (15) and (17) predict the success rate for acquiring 2-dimensional and 1-dimensional targets based on the target bounds. For 1-dimensional targets, the bound is defined by either width or height of the target.

\section{EXPERIMENT}

To assess the accuracy of Equations (15), and (17), we conducted an experiment to evaluate them in both 1-dimensional and 2-dimensional target acquisition tasks.

\section{Participants and Apparatus}

We recruited 12 participants ( 3 females) between ages of 18 and 45. All of them were right-handed and daily mobile device users. The experiment was conducted on a HTC NEXUS 

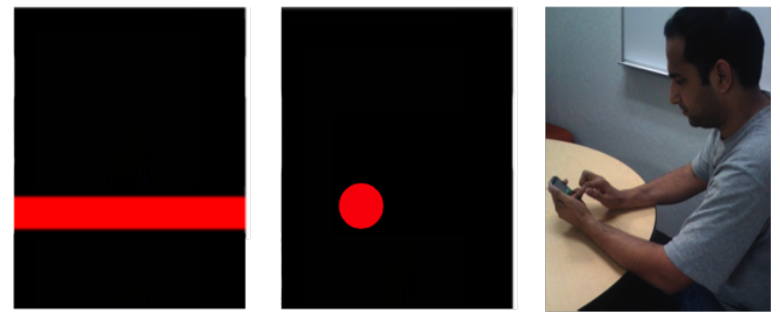

Figure 1. Left: horizontal target in 1-dimensional target acquisition task. Middle: circular target in 2-dimensional target acquisition task. Right: a participant was doing the experiment

smartphone with Android OS. The capacitive touch screen was $48(\mathrm{~mm})$ wide and $80(\mathrm{~mm})$ high with the resolution of $480 \times 800$ pixels.

\section{Design}

The study consisted of both 1-Dimensional and 2Dimensional off-screen-start target acquisition tasks (Figure 1). Each participant held the device using the non-dominant hand and acquired the targets with the index finger of the dominant hand. To simulate the off-screen-start target acquisition condition, participants were asked to rest their dominant hands off the screen in natural and comfortable positions and start from those positions for each trial. Each participant performed the following two tasks:

1-Dimensional Task. At the beginning of each trial, the smartphone played a beep sound and displayed the target as a red bar across the screen (Figure 1 Left). The independent variables were target orientation (vertical or horizontal bar) and target width $(2.4,4.8$ and $7.2 \mathrm{~mm})$. The widths were chosen to reflect the sizes of common UI elements on touchscreens. For example, a hyperlink on a web page is approximately 2.5 mm wide and a key on a smartphone soft keyboard is around 4 $\mathrm{mm}$ wide. Each orientation and width combination included 16 trials which were equally distributed into 2 blocks. The order of trials and locations of targets were randomized. The Interval between every two trials was $1000 \mathrm{~ms}$.
2-Dimensional Task. The procedure was the same with offscreen-start 1-dimensional target acquisition except that a circular target (Figure 1 Middle) rather than a bar target was displayed on the screen. The independent variables were target diameters $(2.4,4.8$, and $7.2 \mathrm{~mm})$.

Participants were advised to acquire the target as quickly and accurately as possible. We used the finger take-off position as the touch location. The target turned yellow and the smartphone played a success beep sound if the touch point hit the inside of the target. Otherwise the target turned blue and played an unpleasant failure sound. The orders of vertical bar, horizontal bar, and circular target acquisition tasks were counterbalanced across the participants.

\section{Results}

We applied Equations (15) and (17) to predict the success rate of target acquisition, and compared the predicted values with observed values.

\section{1-Dimensional Target Acquisition Tasks}

Figures 2 (left) and 3 (left) show the observed and predicted success rates for vertical and horizontal bars separately. As shown, the model performed well for predicting success rates. The differences between observed and predicted success rates were under $5 \%$ for 4.8 and $7.2 \mathrm{~mm}$ targets, and under $10 \%$ for $2.4 \mathrm{~mm}$ targets.

The accurate prediction of success rates was largely due to the accurate prediction of the standard deviations of touch point distributions. Figures 2 (right) and 3 (right) show the observed and predicted standard deviations (predicted via Equation (13)) of touch point distributions. As shown, the predicted values were close to the observed values.

To understand to what degree taking into account the type of input finger will affect the prediction accuracy, we substituted the generic parameters in Equation (17) with the parameters for index finger (Table 1), since all the participants acquired targets with index finger throughout the experiment. Using parameters for index finger has only minor effects on the results. The differences between using two set of parameters

Measures for Acquiring 1-Dimensional Vertical Targets
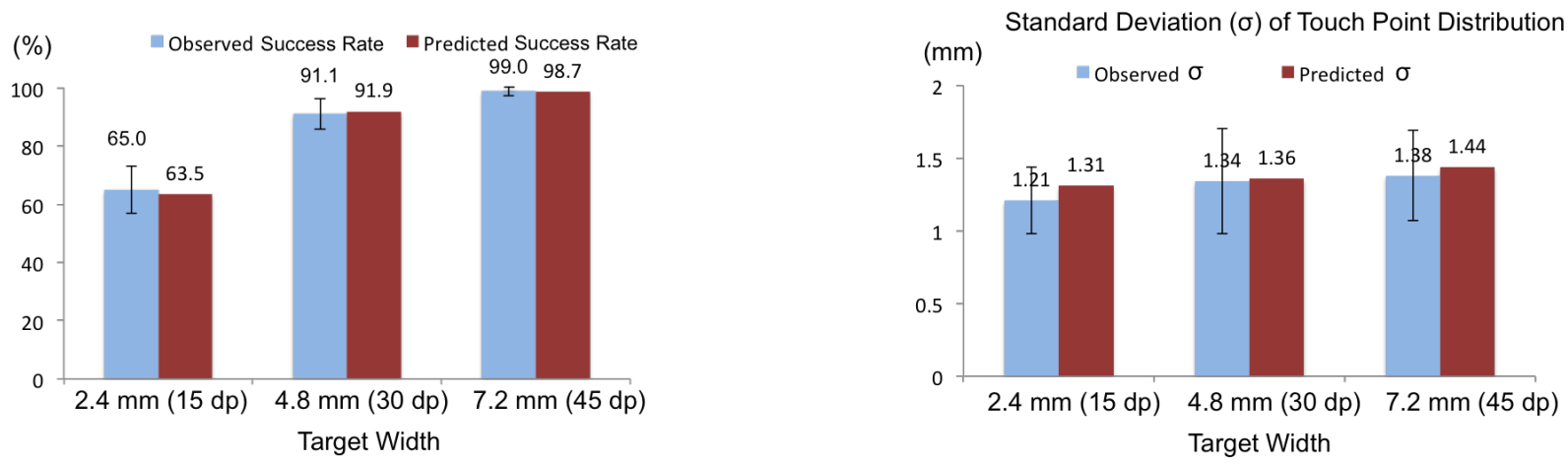

Figure 2. Predicted vs. Observed Measures in 1-Dimensional Vertical Target Acquisition Tasks. Left: Success Rate, Right: Standard Deviation of Touch Point Distribution. Error bars are Std. Dev across participants 

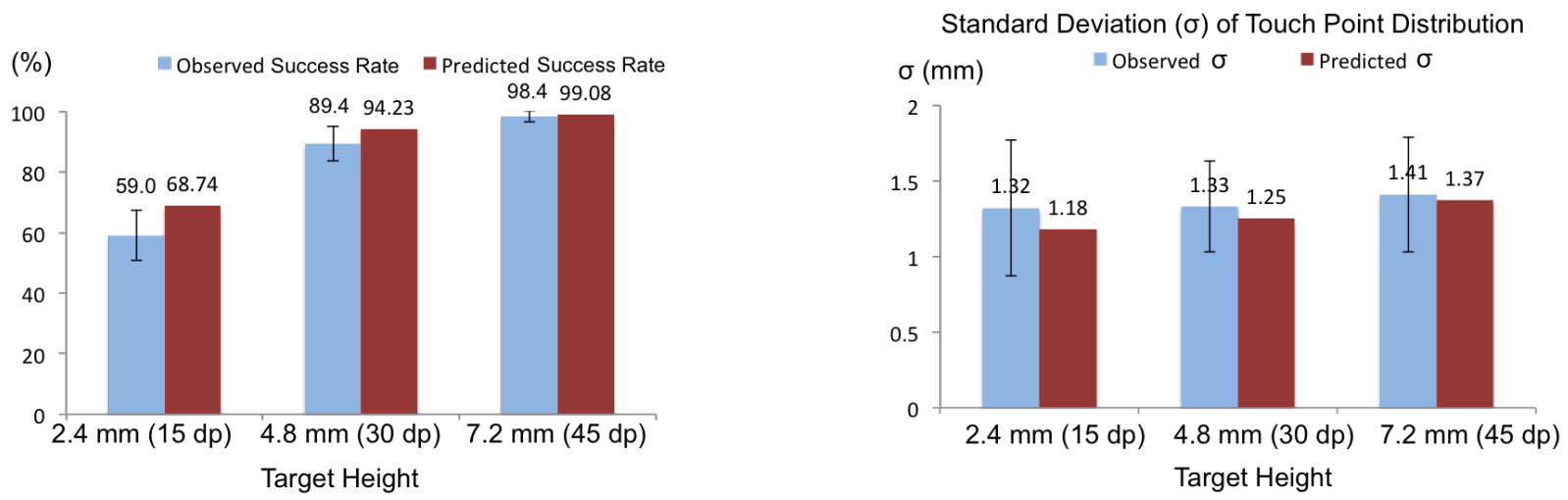

Figure 3. Predicted vs. Observed Measures in 1-Dimensional Horizontal Target Acquisition Tasks. Left: Success Rate, Right: Standard Deviation of Touch Point Distribution. Error bars are Std. Dev across participants

Measures for Acquiring 2-Dimensional Circular Targets
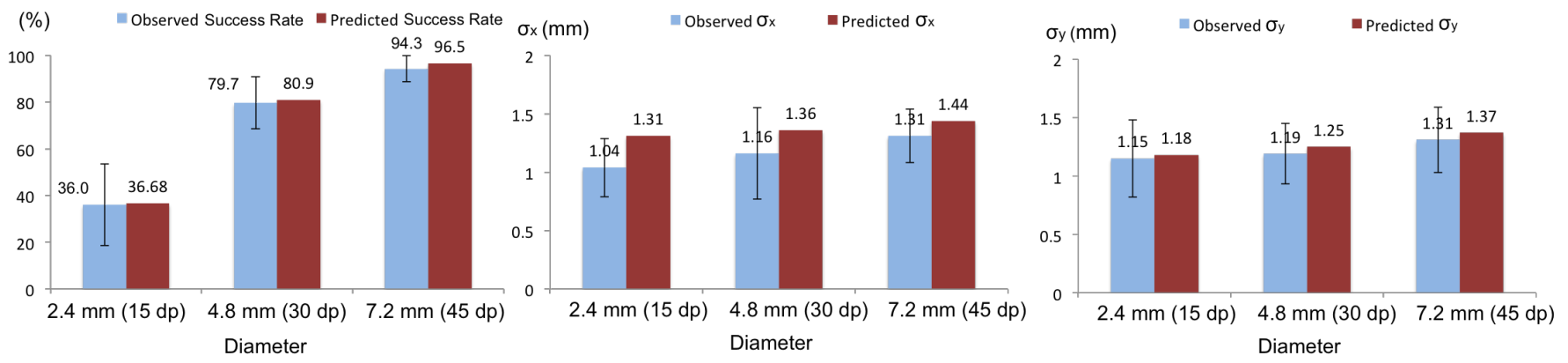

Figure 4. Predicted vs. Observed Measures in 2-Dimensional Circular Target Acquisition Tasks. Left: Success Rate, Middle: Standard Deviation of Touch Point Distribution in $x$ direction, Right: Standard Deviation of Touch Point Distribution in $y$ direction. Error bars are Std. Dev across participants

were less than $2.5 \%$ across all the target size $\times$ target shape combinations.

\section{2-Dimensional Target Acquisition Tasks}

As shown in Figure 4, the predicted success rates were very close to the observed. The observed and predicted success rates were $36.0 \%$ and $36.68 \%$ for $2.4 \mathrm{~mm}, 79.7 \%$ and $80.9 \%$ for $4.8 \mathrm{~mm}$, and $94.3 \%$ and $96.5 \%$ for $7.2 \mathrm{~mm}$. The differences between them were less than $3 \%$.

Figure 4 also shows the observed and predicted standard deviations of touch point distributions in both $x$ and $y$ directions. As shown, the predicted standard deviations of touch point distributions were also close to the observed values.

Similar to 1-dimensional tasks, using index finger parameters (Table 1) had only minor effect compared with using generic parameters. The predicted success rate with generic parameters vs. with index finger parameters were $36.68 \%$ vs. $38.83 \%$ for $2.4 \mathrm{~mm}, 80.9 \%$ vs. $83.03 \%$ for $4.8 \mathrm{~mm}$, and $96.5 \%$ vs. $96.8 \%$ for $7.2 \mathrm{~mm}$ targets.

\section{Overall Prediction Accuracy}

To evaluate the overall prediction accuracy of our model, we plotted the success rates as the (observed, predicted) ordered pairs for each combination of target size $\times$ target shape (Figure 6). A perfect model will place every point on the $y=x$ line. Linear regression results in $y=0.9722 x+4.2382$, with near-unity slope and near-zero intercept, showing a strong model fit.

\section{Discussion}

Our model (Equations (15) and (17)) shows strong prediction accuracy for $4.8 \mathrm{~mm}$ and $7.2 \mathrm{~mm}$ targets: the differences between predicted and observed success rates were under $5 \%$. For the $2.4 \mathrm{~mm}$ targets, the differences were under 10\%. One reason the predicted success rates matched with the observed values well is that its underlying theory, the Dual Gaussian Distribution Model[3, 4], is able to accurately predict the standard deviations of the touch point distributions, as shown in Figures 2, 3 and 4.

Using index-finger parameters has only minor effect on the predicted success rates over using the generic parameters. It is probably because as shown in Bi and Zhai's work [4], although different input fingers affect the spread of touch points, the differences are small. Our findings suggest that using the generic parameters yields reliable prediction. 


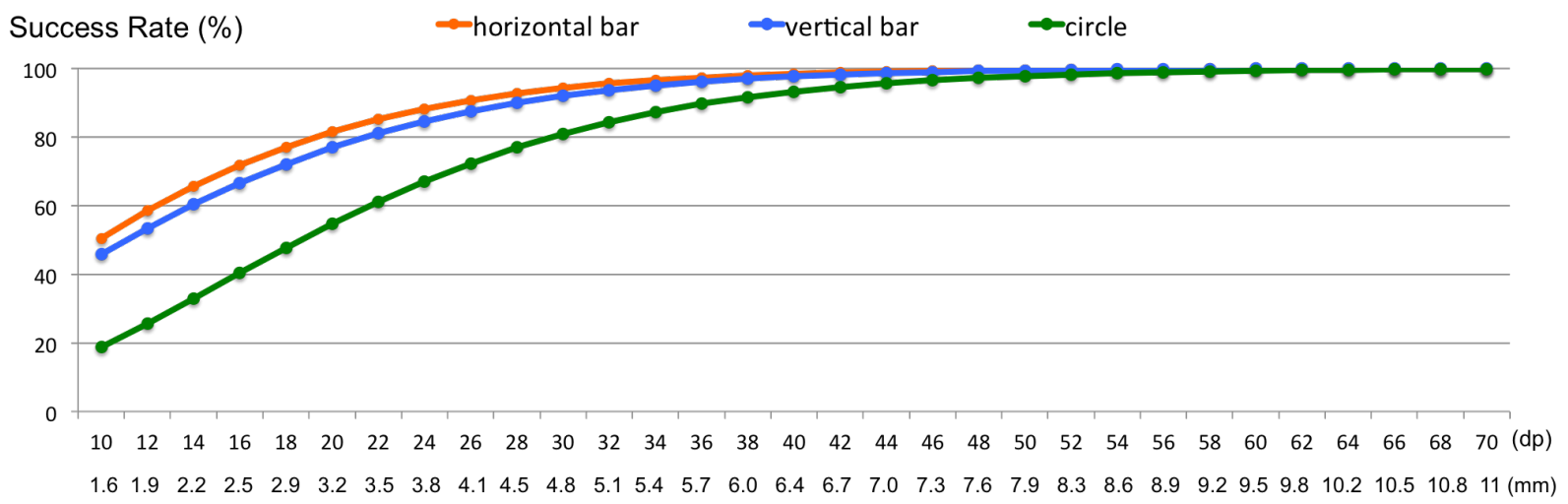

Target Height/Width/Diameter

Figure 5. Predicted Success Rate per Target Size (Height/Width/Diameter) for 1-Dimensional Horizontal and Vertical Targets, and 2-Dimensional Circular Targets.

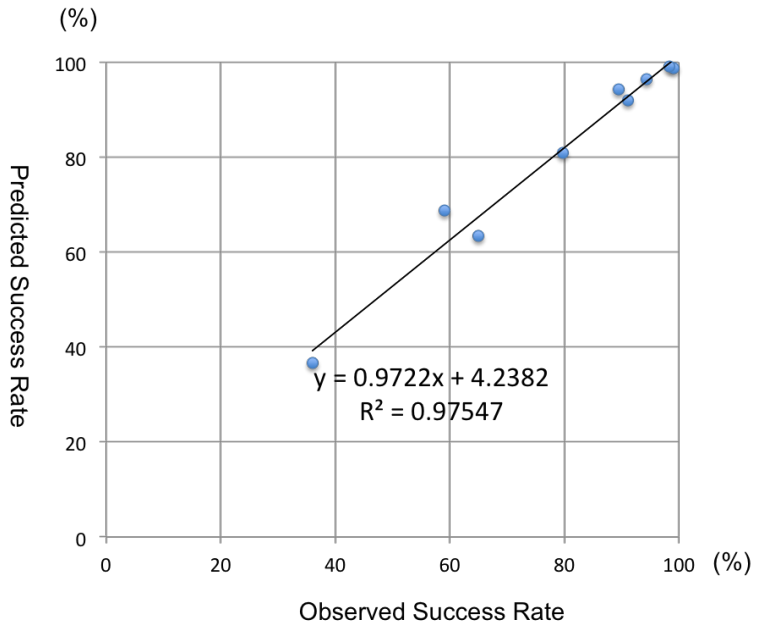

Figure 6. Predicted Success Rates vs. Observed Success Rates for each Target Size $\times$ Target Shape

Our model was created and validated under the assumption that users acquired targets as quickly and accurately as possible. Such a setting reflects just one type of common touchscreen usages, and one of the speed-accuracy conditions. As shown in previous research [16], speed and precision of target acquisition tasks vary depending on the speed-accuracy trade-off strategy the user adopts, which is affected by the experiments instructions, users goal, constraints in real life, etc. We did not focus on these variables in our current investigation. When the effects of these factors can be reliably modelled, they should be incorporated into the model.

\section{APPLYING MODEL TO PREDICT TOUCH ACCURACY}

To provide a guideline on choosing target size, we generate a chart (Figure (5)) showing success rate per target size by applying Equations (15) and (17) over a range of target size. As expected, the success rate increases as the target is becoming bigger. Interestingly, the slopes of all the three curves decrease as the target size increases, indicating that increasing the target size leads to big success rate improvements when the target is small, whereas the gain is diminishing as the target is becoming bigger.

The curves for 1-dimensional targets plateau once the width or height is above $42 \mathrm{dp}(6.7 \mathrm{~mm})$. There is not much performance gain beyond this point. For 2-dimensional target, the curve reaches a plateau at around $48 \mathrm{dp}(7.6 \mathrm{~mm})$.

Figure 5 serves as a tool for choosing the appropriate size for a selectable widget. For example, the recommended minimum height for selectable widgets in Android UI design guideline [1] is $48 \mathrm{dp}$, which has an accuracy of $99.4 \%$ for horizontal bars according to Figure 5. Such a high accuracy indicates that users can comfortably select the target with finger touch. Figure 5 also reveals that $48 \mathrm{dp}$ is already in the plateau region for 1-dimensional target, suggesting that small decreases in the target height will have only minor effect on the accuracy. For example, $40 \mathrm{dp}$ and $38 \mathrm{dp}$ have accuracies of $98.3 \%$ and $97.9 \%$ respectively. Reducing the widget height from $48 \mathrm{dp}$ to these values will cause only minor accuracy degradation. However, it should be noted that our current model parameters and results were based on data collected from relatively stationary conditions. In more mobile such as walking or driving conditions both the model parameters and the model predictions are expected to change.

\section{CONCLUSION}

Building on the Dual Gaussian Distribution Hypothesis[3, 4], we have proposed a simple model that predicts the accuracy of finger-touch target acquisition based on the target size only. An experiment showed that the model made accurate predictions for acquiring 1-dimensional vertical, horizontal, and 2-dimensional circular targets. The differences between predicted and observed success rates were less than $5 \%$ for $4.8 \mathrm{~mm}$ and $7.2 \mathrm{~mm}$ targets, and less than $10 \%$ for 2.4 $\mathrm{mm}$ targets. Such a model will benefit UI designers and researchers in designing and evaluating touchscreen UI, and be instrumental in automatic UI generation systems. 


\section{REFERENCES}

1. Material design for Android, https://www.google.com/design/spec/layout/metricskeylines.htmlmetrics-keylines-touch-target-size.

2. Azenkot, S., and Zhai, S. Touch behavior with different postures on soft smartphone keyboards. In Proceedings of the 14th International Conference on

Human-computer Interaction with Mobile Devices and Services, MobileHCI '12, ACM (New York, NY, USA, 2012), 251-260.

3. Bi, X., Li, Y., and Zhai, S. FFitts law: Modeling finger touch with Fitts' law. In Proceedings of the SIGCHI Conference on Human Factors in Computing Systems, CHI '13, ACM (New York, NY, USA, 2013), 1363-1372.

4. Bi, X., and Zhai, S. Bayesian touch: A statistical criterion of target selection with finger touch. In Proceedings of the 26th Annual ACM Symposium on User Interface Software and Technology, UIST '13, ACM (New York, NY, USA, 2013), 51-60.

5. Fitts, P. M. The information capacity of the human motor system in controlling the amplitude of movement. Journal of Experimental Psychology 47, 6 (1954), 381-391.

6. Henze, N., Rukzio, E., and Boll, S. 100,000,000 taps: Analysis and improvement of touch performance in the large. In Proceedings of the 13th International Conference on Human Computer Interaction with Mobile Devices and Services, MobileHCI '11, ACM (New York, NY, USA, 2011), 133-142.

7. Henze, N., Rukzio, E., and Boll, S. Observational and experimental investigation of typing behaviour using virtual keyboards for mobile devices. In Proceedings of the SIGCHI Conference on Human Factors in Computing Systems, CHI '12, ACM (New York, NY, USA, 2012), 2659-2668.

8. Hick, W. E. On the rate of gain of information. Quarterly Journal of Experimental Psychology 4 (1952), 11-26.
9. Holz, C., and Baudisch, P. The generalized perceived input point model and how to double touch accuracy by extracting fingerprints. In Proceedings of the SIGCHI Conference on Human Factors in Computing Systems, CHI '10, ACM (New York, NY, USA, 2010), 581-590.

10. Holz, C., and Baudisch, P. Understanding touch. In Proceedings of the SIGCHI Conference on Human Factors in Computing Systems, CHI '11, ACM (New York, NY, USA, 2011), 2501-2510.

11. Nichols, J., and Faulring, A. Automatic interface generation and future user interface tools. In Tools ACM CHI 2005 Workshop on The Future of User Interface Design Tools (2005).

12. Schwarz, J., Hudson, S., Mankoff, J., and Wilson, A. D. A framework for robust and flexible handling of inputs with uncertainty. In Proceedings of the 23Nd Annual ACM Symposium on User Interface Software and Technology, UIST '10, ACM (New York, NY, USA, 2010), 47-56.

13. Wang, F., and Ren, X. Empirical evaluation for finger input properties in multi-touch interaction. In Proceedings of the SIGCHI Conference on Human Factors in Computing Systems, CHI '09, ACM (New York, NY, USA, 2009), 1063-1072.

14. Williamson, J. Continuous Uncertain Interaction. $\mathrm{PhD}$ thesis, The University of Glasgow, 2006.

15. Wobbrock, J. O., Cutrell, E., Harada, S., and MacKenzie, I. S. An error model for pointing based on Fitts' law. In Proceedings of the SIGCHI Conference on Human Factors in Computing Systems, CHI '08, ACM (New York, NY, USA, 2008), 1613-1622.

16. Zhai, S., Kong, J., and Ren, X. Speed-accuracy tradeoff in Fitts' law tasks: On the equivalency of actual and nominal pointing precision. Int. J. Hum.-Comput. Stud. 61, 6 (Dec. 2004), 823-856. 